\title{
Talidomida e mieloma múltiplo: verificação dos efeitos terapêuticos através de parâmetros clínico e laboratoriais
} Thalidomide and multiple myeloma: therapy evaluation using clinical and laboratorial parameters

\author{
Rosane Bittencourt ${ }^{1}$ \\ Andreia D. Almeida ${ }^{3}$ \\ Henrique N. S. Bittencourt ${ }^{1}$ \\ Tor Onsten ${ }^{2}$ \\ Flavo Fernandes ${ }^{1}$ \\ João R. Friederich ${ }^{1}$ \\ Laura Fogliato ${ }^{1}$ \\ Claúdia C. Astigarraga ${ }^{1}$ \\ Alessandra Paz \\ Lúcia M. R. Silla
}

\begin{abstract}
Nas duas últimas décadas, houve uma mudança radical na terapia e na evolução do mieloma múltiplo(MM), neoplasia hematológica ainda considerada fatal. As pesquisas e investimentos em medicamentos que interferem com a fisiopatogenia e com o microambiente medular estão permitindo o controle e a regressão do clone plasmocitário maligno, mudando as perspectivas da doença. A idéia nova de usar uma droga velha, a talidomida, tem-se mostrado efetiva no MM. Em 1997, apostando nos efeitos imunomoduladores e antiangiogênicos da talidomida, foram iniciados ensaios clínicos para MM refratários. A partir dai, outras ações sobre o plasmócito e microambiente medular foram eficazes contra a doença, não somente em refratários ou recaídos, mas também como terapia de indução elou de manutenção da remissão. No Serviço de Hematologia do Hospital de Clínicas de Porto Alegre foram acompanhados 35 portadores de mieloma múltiplo, em uso de doses baixas $(100 \mathrm{mg})$ de talidomida, pelas indicações: 13 - manutenção pós-TMO, 11 - pós-indução, 5 -recaída, 4 - refratariedade e 2 - terapia de indução. O estudo vigorou entre março/01 a dez/03. Os parâmetros avaliados foram: nível $\mathrm{Hb}$, pico da imunoglobulina sérica ou urinária e o número de plasmócitos na medula óssea. As medidas foram tomadas pré-talidomida e após 3, 6 e 12 meses. A taxa de imunoglobulina foi o padrão ouro para avaliação de resposta. Os resultados: a dose terapêtica tolerada em 48\% dos pacientes foi $100 \mathrm{mg}$; 65\% dos tratados para induzir remissão (11 pacientes) apresentaram melhora entre $25 \%-50 \%$ no nivel da imunoglobulina sérica; $87,5 \%$ daqueles que usaram para manutenção de remissão (13 pós-TMO/ 11 pós-indução) mantiveram o mesmo plateau inicial. Rev. bras. hematol. hemoter. 2004;26(4):245-255.
\end{abstract}

Palavras-chave: Talidomida; mieloma múltiplo; antiangiogênese.

\section{Introdução}

O mieloma múltiplo (MM) é uma doença maligna caracterizada pela infiltração medular com plasmócitos derivados de um clone anômalo, sustentado pela proemi- nente angiogênese, que se ancora nos fatores estimulantes de crescimento do endotélio e dos fibroblastos, associados à ação das moléculas de adesão, das interleucinas 6 (IL-6), IL-3, IL-1 $\beta$ e IL-10, dos fatores estimulantes do crescimento de granulócitos e monócitos e do fator de

\footnotetext{
Médico do Serviço de Hematologia e Transplante de Medula Óssea do Hospital de Clínicas de Porto Alegre.

Professor do Departamento de Medicina Interna - UFRGS.

${ }_{3}^{3}$ Médico Residente do Serviço de Hematologia e Transplante de Medula Óssea do Hospital de Clínicas de Porto Alegre.

${ }^{4}$ Professora do Departamento de Medicina Interna - UFRGS e Chefe do Serviço de Hematologia e Transplante de Medula Óssea do Hospital de Clínicas de Porto Alegre.
}

Correspondência para: Rosane Bittencourt

Rua Ramiro Barcelos, 2350 - Rio Branco

Hospital das Clínicas Porto Alegre - Serviço de Hematologia Clínica - Sala 2235

90035-003 - Porto Alegre-RS-E-mail: rosane@sms.prefpoa.com.br 
necrose tumoral alfa (TNF- $\alpha){ }^{1}$ Pela acentuada angiogênese, o MM tem sido o protótipo para o estudo de terapias capazes de interferir com a densidade da vasculatura do microambiente medular e, conseqüentemente, diminuir o estímulo para estas células malignas. ${ }^{2}$

O MM, neoplasia invariavelmente fatal, tem incidência de 4/100.000 pessoas/ano, ocupando cerca de $15 \%$ das doenças hematológicas malignas, sendo responsável por $2 \%$ das mortes decorrentes de câncer em um ano nos Estados Unidos. ${ }^{3}$ É uma doença sistêmica, com sintomatologia variável onde se destacam as dores ósseas, os sintomas secundários à anemia, os distúrbios metabólicos e renais, passíveis de gerar incapacidade física e emocional pelo nível acentuado de comprometimento do indivíduo. Quando suspeitado pelo quadro clínico e laboratorial, facilmente chega-se ao diagnóstico, lançando aí o desafio da escolha de um tratamento eficiente. Os tratamentos convencionais produzem remissões satisfatórias, porém temporárias, e a doença segue seu curso fatal se não forem introduzidas medidas para a supressão do clone maligno. Os melhores esquemas quimioterápicos promovem até $90 \%$ de remissões, porém permanecem sem modificar o tempo de sobrevivência. ${ }^{4}$ Antes das terapias de altas doses com resgate de células-tronco hematopoéticas (auto-transplante), aproximadamente $25 \%$ dos pacientes sobreviviam cinco anos, e menos de $10 \%$ atingiam dez anos de sobrevida. ${ }^{4,5}$ Hoje, felizmente, o autotransplante seguido das estratégias de manutenção e suporte estão mudando este cenário, permitindo longas sobrevidas com melhor bem-estar. Considerando que uma grande parte dos pacientes, quando do diagnóstico, estão numa fase da vida voltados para atividades produtivas, é mister a busca de novas medidas terapêuticas no sentido de melhorar induções de remissão e prolongar os períodos de respostas. ${ }^{6}$ As pesquisas têm-se centrado no reconhecimento de mecanismos imunorregulatórios envolvidos na patogênese do mieloma. $\mathrm{O}$ fato de que o tumor requer a neovascularização para crescer tem aberto novos caminhos para os agentes antiangiogênicos, incluindo-se aí a talidomida, que inibe os fatores estimulantes do endotélio e do fibroblasto, responsáveis pela neovascularização e, desta maneira, impedem o tumor de crescer e metastatizar. ${ }^{2,7,8}$

Em 1997, apostando nos efeitos imunomoduladores e antiangiogênicos da talidomida, foram iniciados ensaios clínicos para tratamentos de MM refratários. A partir daí, outras ações sobre o plasmócito e microambiente medular foram efetivos para controlar a doença. Nos primeiros estudos foram incluídos: a) portadores de mieloma com fatores predisponentes de pobre prognóstico, como deleção do cromossomo $13, \mathrm{~b}$ ) resistentes a esquemas prévios de poliquimioterapias, e c) recaídos após autotransplante. Os resultados foram favoráveis nos três grupos, com redução de índices da atividade da doença em até $75 \%$, e respostas completas em $90 \%$ dos casos. Este sucesso tera- pêtico fez com que a atividade da talidomida fosse melhor estudada, sendo comprovada sua ação antitumoral através de vários mecanismos, destacando-se o efeito antiangiogênico, a ação anti-IL-6, estímulo da apoptose, a supressão de fator de necrose tumoral alfa e a expansão de efeitos imunomodulatórios com a produção do interferon gama e IL-2.,

Em 1999, é reconhecido o papel da talidomida na Hematologia, representando um novo e importante agente no controle do mieloma em pacientes sem condições de terapias intensivas e/ou nos recaídos após o tratamento poliquimioterápico convencional.

Nos anos 2000, está justificado e consolidado o uso da talidomida na rotina de tratamento do MM. ${ }^{9}$ No Brasil, a Agência Nacional de Vigilância Sanitária (Anvisa) liberou, através da Resolução n³4, de 20 de abril de 2000, a utilização de talidomida no tratamento de MM refratários à quimioterapia. Posteriormente, a Portaria Conjunta ${ }^{\circ} 25$, da Secretaria de Políticas de Saúde, publicada em janeiro/02, regulamentou seu uso.

Em 33 meses, acompanhamos um grupo de pacientes com respostas clínicas interessantes de serem divulgadas, não só corroborando as notificações contemporâneas da literatura, ${ }^{9,10,11}$ mas também como fonte multiplicadora de opções terapêuticas para o MM quando falha às terapias iniciais ou mesmo como manutenção de um plateau preestabelecido.

\section{Casuística e Métodos}

No presente estudo, a equipe da Hematologia do Hospital de Clínicas de Porto Alegre acompanhou prospectivamente 35 pacientes com diagnóstico de mieloma múltiplo que receberam talidomida. Esta medicação é distribuída gratuitamente pela Secretaria de Saúde do Estado-RS. O estudo iniciou em março/01, estendendo-se até dezembro de 2003. Neste período, os pacientes realizaram exames laboratoriais e consultas clínicas mensalmente. Todos realizaram os exames laboratoriais de rotina, descritos na tabela 1 , na véspera da consulta e receberam simultaneamente o tratamento de suporte padronizado no Serviço de Hematologia descrito na tabela 2. As principais indicações para a introdução de talidomida estão detalhadas na tabela 3. Na tabela 4, encontra-se o esquema de tratamento desenhado pelo Serviço de Hematologia, baseado nas indicações da literatura. ${ }^{9,10,11} \mathrm{O}$ plano previa a dose inicial de $200 \mathrm{mg}$ de talidomida durante trinta dias, seguido de nova avaliação clínica e laboratorial. O objetivo seria aumentar a dose progressivamente até atingir 400 mg. Os exames para avaliação da efetividade do uso da talidomida, tabela 5 , foram realizados em todos os pacientes em três momentos: pré-talidomida, três e seis meses após a terapia. No final do estudo, 14 pacientes estavam usando talidomida há mais de um ano e fizeram uma quarta 
avaliação. Nos pacientes que receberam talidomida como manutenção de remissão foi considerado efeito terapêutico o plateau, ou seja a estabilização dos marcadores de resposta: a hemoglobina, número de plasmócitos na medula óssea, pico monoclonal e quantidade de imunoglobulina envolvida. Naqueles em que a talidomida foi usada devido a recaídas, refratariedade ou indução de remissão, o efeito terapêutico foi definido como redução nos níveis de

Tabela 1

Exames laboratoriais rotina mensal

Hemograma
Plaquetas
Creatinina
TGO/TGP/Bilirrubinas
Cálcio

Tabela 2

Tratamento de suporte no mielomamúltiplo

Inibidor de osteólise $=$ pamidronato $-90 \mathrm{mg} /$ mensal $-\mathrm{ev}-2 \mathrm{~h}$

Cálcio + vitamina D - vo diariamente

Folin - $5 \mathrm{mg}$ se $\mathrm{hb}<10 \mathrm{~g}$ e macrocitose

Bactrim $3 \times$ semana e acilovir diariamente durante qt

Tabela 3

Indicações para talidomida

\begin{tabular}{lcc}
\hline Indicação & Legendas & $\begin{array}{c}\mathrm{N}^{\circ} \text { de } \\
\text { Pacientes }\end{array}$ \\
\hline Terapia pós-indução da remissão & $\mathrm{A}$ & 11 \\
Manutenção pós-auto TMO & $\mathrm{B}$ & 13 \\
Recaída após 2 esquemas & $\mathrm{C}$ & 05 \\
$\quad$ qumioconvencional & $\mathrm{D}$ & 04 \\
Refratariedade & $\mathrm{E}$ & 02 \\
Indução (1 (1) linha) $^{\text {Total }}$ & & 35 \\
\hline
\end{tabular}

Tabela 4

Desenho inicial do estudo - talidomida - HCPA

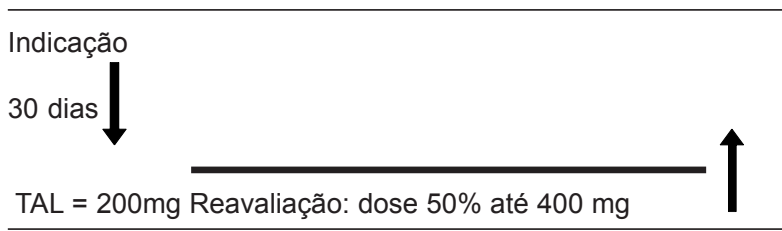

Tabela 5

Exames para avaliação talidomida

\begin{tabular}{lcccc}
\hline Exames & \multicolumn{4}{c}{ Tempo dos exames (meses) } \\
\hline & Início Tal & $3 \mathrm{M}$ & $6 \mathrm{M}$ & $12 \mathrm{M}$ \\
\hline Hemoglobina & $\mathrm{X}$ & $\mathrm{X}$ & $\mathrm{X}$ & $\mathrm{X}$ \\
$\begin{array}{c}\text { Proteinograma } \\
\text { sérico ou urinário }\end{array}$ & $\mathrm{X}$ & $\mathrm{X}$ & $\mathrm{X}$ & $\mathrm{X}$ \\
$\begin{array}{c}\text { Imunoeletroforeses } \\
\text { sérica ou urinária }\end{array}$ & $\mathrm{X}$ & $\mathrm{X}$ & $\mathrm{X}$ & $\mathrm{X}$ \\
$\begin{array}{c}\text { Melograma } \\
\left(\mathrm{N}^{\circ} \text { plasmócitos }\right)\end{array}$ & $\mathrm{X}$ & $\mathrm{X}$ & $\mathrm{X}$ & $\mathrm{X}$ \\
\hline
\end{tabular}

imunoglobulina sérica ou urinária conforme os critérios de Bladè. ${ }^{6}$

Trinta e um pacientes $(88,5 \%)$ iniciaram o esquema terapêutico com a dose de $200 \mathrm{mg} /$ noite, conforme o preconizado; quatro (11,5\%) iniciaram com $100 \mathrm{mg}$ : dois devido à idade maior de 70 anos e comorbidades e dois por hipertensão e diabetes respectivamente. Em cada consulta mensal, a dose foi reavaliada considerando os efeitos colaterais e terapêuticos. Em seis meses, 14 pacientes (48\%) que iniciaram com $200 \mathrm{mg}$ estavam usando metade da dose (100 mg). Somente em três pacientes a dose foi progressivamente aumentada, e apenas um destes tolerou $300 \mathrm{mg}$ como dose efetiva. Em quatro pacientes, a dose terapêutica aceitável foi $50 \mathrm{mg}$. As doses foram individualizadas para cada paciente, respeitando a tolerância de cada um. Os comportamentos clínico e laboratorial dos pacientes estudados serão apresentados na seção de resultados descritas a seguir.

\section{Resultados}

Este estudo informa o seguimento de um grupo de pacientes, portadores de mieloma múltiplo, em uso de talidomida durante o período de março/2001 até dezembro/03, quando as respostas clínicas foram avaliadas. As características dos pacientes e as imunoglobulinas envolvidas nos diagnósticos de mieloma estão descritas nas tabelas 6 e 7 respectivamente.

Em 2001, 5/35 pacientes entraram no estudo e permanecem até dez/03. Em 2002, 9/35 iniciaram a talidomida e 3/9 tiveram a medicação suspensa definitivamente após 6 , 10 e 16 meses respectivamente, devido aos efeitos colaterais. O primeiro paciente apresentou arritmia cardíaca com fibrilação atrial e trombose venosa profunda (TVP) em membro inferior esquerdo; o segundo, vertigens incontroláveis, e o terceiro, hepatopatia pelo vírus C. A maioria - 21/35 pacientes - iniciou a talidomida no decorrer do ano 2003, estando há menos de um ano de

Tabela 6

Características dos pacientes

\begin{tabular}{lc}
\hline Características & $N^{\circ}$ de pacientes \\
\hline Sexo M/F & $14 \mathrm{~m} / 21 \mathrm{f}=1 \mathrm{~m} / 1,5 \mathrm{f}$ \\
Idade (mediana) & 59 anos \\
Etnia B/N & 26 brancos $/ 9$ negros
\end{tabular}

Tabela 7

Imunoglobulinas envolvidas

\begin{tabular}{lc}
\hline Imunoglobulinas & $N^{\circ}$ de pacientes \\
lgg & 23 \\
lga & 06 \\
Cadeias leves & 05 \\
Não secretor & 01 \\
\hline Total & 35 \\
\hline
\end{tabular}


Tabela 8

Panorama geral sobre dose, o tempo de uso em ordem cronológica e os paraefeitos da talidomida em cada paciente

\begin{tabular}{|c|c|c|c|c|c|c|c|c|}
\hline Paciente & Sexo & Idade & Indicação & $\begin{array}{l}\text { Data } \\
\text { início }\end{array}$ & Tempo & $\begin{array}{l}\text { Dose } \\
\text { inicial }\end{array}$ & $\begin{array}{l}\text { Dose } \\
\text { atual }\end{array}$ & Paraefeitos \\
\hline 1 & $M$ & 51 & B & 03/2001 & $33 \mathrm{~m}$ & 200 & 100 & 7 \\
\hline 2 & $\mathrm{~F}$ & 69 & $A$ & $05 / 2001$ & $31 \mathrm{~m}$ & 200 & 100 & 1 \\
\hline 3 & $\mathrm{~F}$ & 84 & A & $05 / 2001$ & $31 \mathrm{~m}$ & 200 & 50 & $1,2,8$ \\
\hline 4 & $\mathrm{~F}$ & 78 & $A$ & $05 / 2001$ & $31 \mathrm{~m}$ & 200 & 100 & 1,7 \\
\hline 5 & $\mathrm{~F}$ & 49 & C & $11 / 2001$ & $25 \mathrm{~m}$ & 200 & 100 & $1,2,4$ \\
\hline 6 & $\mathrm{~F}$ & 67 & A & $03 / 2002$ & $21 \mathrm{~m}$ & 100 & 200 & Nenhum \\
\hline 7 & $\mathrm{~F}$ & 75 & $A$ & $03 / 2002$ & $21 \mathrm{~m}$ & 200 & 150 & 2,9 \\
\hline 8 & $M$ & 50 & $B$ & $05 / 2002$ & $16 \mathrm{~m}$ & 200 & ------ & SUSP DEF 1 \\
\hline 9 & M & 56 & A & $07 / 2002$ & $17 \mathrm{~m}$ & 200 & 100 & $1,2,4$ \\
\hline 10 & $F$ & 74 & $C$ & $08 / 2002$ & $10 \mathrm{~m}$ & 200 & --.- & SUSP DEF 2 \\
\hline 11 & $M$ & 66 & $A$ & 09/2002 & $15 \mathrm{~m}$ & 200 & 200 & Nenhum \\
\hline 12 & $\mathrm{~F}$ & 68 & $A$ & $10 / 2002$ & $14 \mathrm{~m}$ & 200 & 200 & Nenhum \\
\hline 13 & $\mathrm{~F}$ & 54 & B & $11 / 2002$ & $13 \mathrm{~m}$ & 200 & 100 & 7 \\
\hline 14 & $M$ & 72 & $D$ & $12 / 2002$ & $6 m$ & 100 & -ב-- & SUSP DEF 3 \\
\hline 15 & M & 47 & B & $01 / 2003$ & $11 \mathrm{~m}$ & 200 & 200 & Nenhum \\
\hline 16 & $F$ & 72 & A & $02 / 2003$ & $10 \mathrm{~m}$ & 200 & 50 & 7,9 \\
\hline 17 & $\mathrm{~F}$ & 57 & B & $03 / 2003$ & $9 \mathrm{~m}$ & 200 & 100 & 1 \\
\hline 18 & $\mathrm{~F}$ & 62 & C & $03 / 2003$ & $9 \mathrm{~m}$ & 200 & 300 & Nenhum \\
\hline 19 & $\mathrm{~F}$ & 68 & $A$ & $03 / 2003$ & $9 \mathrm{~m}$ & 200 & 100 & $1,2,5,9$ \\
\hline 20 & M & 79 & E & $03 / 2003$ & $9 \mathrm{~m}$ & 200 & 100 & 1,4 \\
\hline 21 & M & 55 & B & $04 / 2003$ & $8 \mathrm{~m}$ & 200 & 50 & 2,5 \\
\hline 22 & M & 54 & B & $04 / 2003$ & $8 \mathrm{~m}$ & 200 & 100 & $1,2,9$ \\
\hline 23 & M & 65 & A & $05 / 2003$ & $7 \mathrm{~m}$ & 200 & 150 & $1,2,9$ \\
\hline 24 & M & 59 & $\mathrm{D}$ & $05 / 2003$ & $7 \mathrm{~m}$ & 200 & 100 & $1,2,5,9$ \\
\hline 25 & $\mathrm{~F}$ & 72 & $A$ & $06 / 2003$ & $6 \mathrm{~m}$ & 200 & 100 & $2,3,9$ \\
\hline 26 & $\mathrm{~F}$ & 66 & $\mathrm{C}$ & $06 / 2003$ & $6 \mathrm{~m}$ & 200 & 100 & $1,2,9$ \\
\hline 27 & $\mathrm{~F}$ & 54 & B & $06 / 2003$ & $6 \mathrm{~m}$ & 200 & 200 & Nenhum \\
\hline 28 & $M$ & 69 & $A$ & $06 / 2003$ & $6 \mathrm{~m}$ & 200 & 100 & 1,9 \\
\hline 29 & $M$ & 46 & $B$ & 07/2003 & $5 \mathrm{~m}$ & 200 & 100 & $1,5,6$ \\
\hline 30 & $\mathrm{~F}$ & 53 & B & $08 / 2003$ & $4 \mathrm{~m}$ & 200 & 200 & Nenhum \\
\hline 31 & $M$ & 40 & B & $09 / 2003$ & $3 \mathrm{~m}$ & 200 & 200 & Nenhum \\
\hline 32 & $\mathrm{~F}$ & 65 & A & $09 / 2003$ & $3 \mathrm{~m}$ & 200 & 150 & 1,5 \\
\hline 33 & $\mathrm{~F}$ & 54 & B & $09 / 2003$ & $3 \mathrm{~m}$ & 200 & 100 & 9 \\
\hline 34 & $\mathrm{~F}$ & 74 & $D$ & $10 / 2003$ & $2 \mathrm{~m}$ & 100 & 50 & 1,2 \\
\hline 35 & $\mathrm{~F}$ & 72 & E & $11 / / 2003$ & $1 \mathrm{~m}$ & 100 & 100 & Nenhum \\
\hline
\end{tabular}

Indicação uso da talidomida: $A=$ terapia pós-químio de indução; $B=$ manutenção plateau pós-TMO autólogo; $C$ = recaída após esquemas químio; $D=$ refratariedade; $E=$ indução 1 linha.

Paraefeitos: Parestesias extremidades $=1$; tonturas $=2$; vertigens $=3$; constipação =4; Secura da boca $=5$; rash =6; zumbidos =7; edema MMII =8; sonolencia = 9. Motivos da suspensão definitiva de talidomida: Susp def $1=$ hepatopatia virus C; Susp def 2 =vertigens; Susp def $3=$ fibrilação atrial e TVP tratamento no momento da descrição destes dados.

Na tabela 8 estão expostos a indicação, a data de início do tratamento em ordem cronológica, o tempo de uso, as doses inicial e atual, e os efeitos apresentados por cada paciente com a talidomida. Observando esta tabela verifica-se que: 1) a principal indicação foi a manutenção de remissão pós-transplante autólogo, ou pósindução com quimioterapia (24/35 pacientes), recaídas, refratariedade e terapia de $1^{\text {a }}$ linha foi motivo nos demais 11 pacientes; 2 ) a maioria, $23 / 35$ pacientes, necessitou redução na dose inicial no decorrer do estudo; 3 ) a dose de $100 \mathrm{mg}$ foi tolerada por $17 / 35$; 4) $9 /$ $35(25,5 \%)$ não apresentaram efeitos colaterais. O comportamento das doses está ilustrado na tabela 9 , onde se verifica que o esquema de doses previsto inicialmente não foi efetivado, porque 23/35 (65\%) dos pacientes não toleraram os $200 \mathrm{mg}$, devido aos paraefeitos mencionados, necessitando redução de $25 \%$ a $75 \%$ das doses prescritas no início do estudo. O gráfico 1 mostra a freqüência das doses usadas durante o período estudado. A dose de $100 \mathrm{mg}$ foi considerada padrão, atingindo efeitos terapêuticos com paraefeitos controlados em 17/35 pacientes. Os principais paraefeitos apresentados neste grupo e as medidas adotadas para amenizá-los estão descritos na tabela 10 . Nos 11 pacientes que usaram a talidomida para induzir remissão de doença, a resposta clínica foi aferida através da taxa de imunoglobulina, e 7/11(65\%) apresentaram reduções entre $25 \%$ a $50 \%$. Os parâmetros de resposta podem ser visualizados na tabela 11, onde estão os dados pré e pós-talidomida.

\section{Discussão}

$\mathrm{O}$ fato de a angiogênese estar nitidamente aumentada em hematopatias como o $\mathrm{MM}^{12,13}$ faz as drogas antiangiogênicas, como a talidomida, representar uma nova possibilidade de abordagem menos tóxica para o seu 
tratamento. Pesquisadores como Singhal, Rajukmar, Barlogie, entre outros, ${ }^{7,9,10,11,14}$ demonstraram a atividade da talidomida em portadores de MM, como terapia de indução em primeira linha ou como terapia de salvamento. Enquanto nos trabalhos revisados ${ }^{7,8,15,16}$ as principais indicações da talidomida no MM foram os casos de recaída

Tabela 9

Comportamento das doses toleradas

$17 / 35=(48,5 \%)$ dos pacientes redução dose $50 \%$

$07 / 35=(20 \%)$ mantiveram mesma dose inicial

$03 / 35=(8,5 \%)$ pacientes redução dose $75 \%$

$03 / 35=(8,5 \%)$ pacientes redução dose $25 \%$

$02 / 35=(6 \%)$ pacientes aumento dose $=1 \mathrm{em} 50 \%$ e $1 \mathrm{em} 100 \%$ $03 / 35=(8,5 \%)$ suspensão definitivamente

Gráfico 1

Representação das freqüências das doses da talidomida

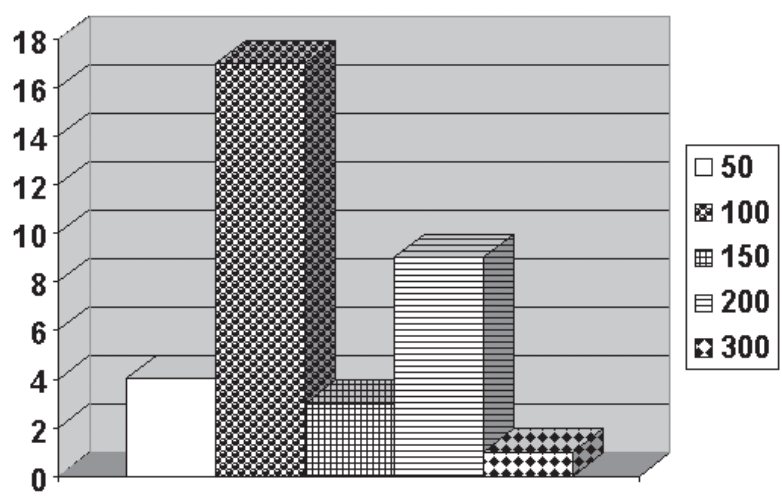

Tabela 10

Efeitos colaterais e as medidas

\begin{tabular}{|c|c|c|}
\hline Efeito Colateral & Pacientes & Manejo \\
\hline Nenhum & 9 & \\
\hline TVP/ACFA & 1 & Suspensão definitiva da Tal \\
\hline $\begin{array}{l}\text { Parestesias } \\
\text { Extremidades }\end{array}$ & 16 & $\begin{array}{c}\text { Redução de } 50 \% \text { da dose e } \\
\text { e introdução do } \\
\text { complexo vitamínico B }\end{array}$ \\
\hline Tontura & 13 & $\begin{array}{l}\text { Suspensão temporária da Tal } \\
\text { e reintrodução com } 50 \% \text { da dose }\end{array}$ \\
\hline Sonolência & 10 & $\begin{array}{l}\text { Suspensão temporária da Tal } \\
\text { e reintrodução com } 50 \% \text { da dose }\end{array}$ \\
\hline Boca seca & 5 & $\begin{array}{c}\text { Redução de } 50 \% \text { da dose e } \\
\text { umidificadores orais }\end{array}$ \\
\hline Constipação & 4 & $\begin{array}{l}\text { Emolientes fecais e } \\
\text { dieta com fibras }\end{array}$ \\
\hline Edema MMII & 3 & Redução de $50 \%$ da dose \\
\hline Zumbidos & 3 & Redução de $50 \%$ da dose \\
\hline Vertigens & 1 & Suspensão definitiva da Tal \\
\hline Rash & 1 & $\begin{array}{l}\text { Creme de aveia e } \\
\text { anti-histamínicos }\end{array}$ \\
\hline
\end{tabular}

Alteração da função hepática $e$ vírus $\mathrm{C}$ positivo e refratariedade, neste estudo local, a principal indicação tem sido a terapia pós-tmo autólogo ou pós-quimioterapia de indução, semelhantes às indicações citadas por Rajkumar ${ }^{9}$ e Barlogie ${ }^{17}$ em algumas publicações.

Após acompanhar este grupo durante 33 meses, as principais observações foram: 1) pelas indicações de indução de remissão para recaída, refratariedade ou primeira linha, $65 \%$ dos pacientes apresentaram redução dos marcadores de atividade de doença em até seis meses de uso; 2) naqueles cuja indicação foi manter o plateau, 87,5\% atingiram o objetivo; 3) verificou-se que a dose de $100 \mathrm{mg}$ é perfeitamente efetiva com um tempo médio de 12 semanas para apresentar resposta satisfatória, repetindo os resultados das experiências publicadas por Larkin ${ }^{18} \mathrm{e}$ Palumbo, ${ }^{19}$ onde afirmam que as doses de $100 \mathrm{mg}$ ou mesmo 50 mg de talidomida são bem toleradas e efetivas contra as células do MM.

Entre os paraefeitos apresentados destacam-se: parestesias em extremidades 16/35 (46\%); tonturas 13/35 (37\%); sonolência 10/35 (28\%); boca seca 5/35 (14\%) e constipação 4/35 (11\%). Em 9/35 (26\%) não foram descritos efeitos colaterais. Três pacientes apresentaram intercorrências graves: trombose venosa profunda e fibrilação atrial, hepatotoxicidade associada ao vírus $\mathrm{C}$ da hepatite $\mathrm{e}$ tonturas intoleráveis, que impediram a continuidade do tratamento. Todos os paraefeitos manifestados por este grupo estão de acordo com os efeitos colaterais descritos na literatura. ${ }^{20,21,22}$

Na série acompanhada, os benefícios da talidomida foram clínica e laboratorialmente satisfatórios, servindo de paradigma para a utilização desta na rotina do atendimento dos portadores de mieloma múltiplo, confirmando que é possível obter resposta clínica e hematológica sem mielossupressão e com paraefeitos controláveis.

Atualmente, no Serviço de Hematologia e Transplante Autólogo de Medula Óssea do HC Porto Alegre, a talidomida tem sido instituída como terapia de manutenção pós-autólogo, ou como alternativa terapêutica em recidivas ou refratariedade à quimioterapia, ou ainda como esquema de indução nos pacientes acima de 80 anos. A dose ideal de talidomida no mieloma ainda não está definitivamente estabelecida; enquanto os trabalhos mais clás$\operatorname{sicos}^{14,17,20,23}$ referem doses de $200 \mathrm{mg}$ até $800 \mathrm{mg}$, várias publicações preconizam doses inferiores aos $200 \mathrm{mg}$. ${ }^{18,19,24}$ Os resultados apresentados nesta amostra apontam 100 mg como dose efetiva, deixando ainda uma perspectiva de aumentar progressivamente conforme a tolerância, efeitos colaterais e as respostas clínicas e laboratoriais.

\section{Talidomida: aspectos da literatura}

\section{Aspecto histórico}

A talidomida é um derivado do ácido glutâmico, cuja fórmula ativa consiste em ([N-pphtthalimido] - gluta- 
Tabela 11

Comparação de parâmetros de doença pré e pós-talidomida

\begin{tabular}{|c|c|c|c|c|c|c|c|c|c|c|c|}
\hline \multirow[t]{2}{*}{ Paciente } & \multirow[t]{2}{*}{$\operatorname{lnd}^{*}$} & \multirow[t]{2}{*}{ Dose 1} & \multirow[t]{2}{*}{ Dose 2} & \multicolumn{4}{|c|}{ Exames Pré } & \multicolumn{4}{|c|}{ Exames 6 meses Pós } \\
\hline & & & & $\mathrm{Hb}$ & Pico $M^{* *}$ & $\lg$ & Plas MO & $\mathrm{Hb}$ & Pico M & $\lg$ & Plas MO \\
\hline 1 & B & 200 & 100 & 12 & $\gamma 1,9$ & G 2000 & 6 & 12,5 & $\gamma 1,3$ & G 1600 & 2 \\
\hline 2 & A & 200 & 100 & 10,8 & $\beta 2,5$ & A 1720 & 12 & 11,5 & $\beta 2,0$ & A 990 & 2 \\
\hline 3 & A & 200 & 50 & 12 & $\gamma 2,66$ & G 3030 & 10 & 12,9 & $\gamma 1,7$ & G 1950 & 5 \\
\hline 4 & A & 200 & 100 & 11,4 & $\gamma 2,9$ & G 3690 & 13 & 12,5 & $\gamma 2,1$ & G 2080 & 5 \\
\hline 5 & $\mathrm{C}$ & 200 & 100 & 8,5 & $\begin{array}{l}\text { Soro } \\
\kappa=2740 \\
\lambda=1590\end{array}$ & $\begin{array}{l}\text { Urin } \\
\mathrm{k}=61 \\
\mathrm{~g}=43\end{array}$ & 7 & 10,5 & $\begin{array}{l}\text { Soro } \\
\kappa=2850 \\
\gamma=1400\end{array}$ & $\begin{array}{l}\text { Urin } \\
k=59 \\
q=40\end{array}$ & 3 \\
\hline 6 & B & 100 & 200 & 12,1 & $\beta 1,53$ & A 410 & 5 & 13,8 & $\beta 1,13$ & A 187 & 1 \\
\hline 7 & A & 200 & 150 & 10,9 & $\gamma 1,3$ & G 2700 & 10 & 9 & $\gamma 1,03$ & G 1030 & 2 \\
\hline 8 & B & 200 & ---- & 8,3 & $\gamma 1,34$ & G 2100 & 9,2 & 11 & $\gamma 1,1$ & G 1390 & 7 \\
\hline 9 & A & 200 & 100 & 11 & $\gamma 1,9$ & G 3050 & 12 & 12,9 & $\gamma 1,3$ & G 2700 & 7 \\
\hline 10 & C & 200 & ---- & 9,0 & $\gamma 4,89$ & G 6030 & 21 & 9,7 & $\gamma 3,06$ & G 3500 & 10 \\
\hline 11 & A & 200 & 200 & 12 & $\gamma 1,9$ & G 1560 & 8 & 12,6 & $\gamma 1,01$ & G 1300 & 5 \\
\hline 12 & A & 200 & 200 & 9,3 & $\begin{array}{l}\text { Soro } \\
\kappa=2100\end{array}$ & $\begin{array}{l}\text { Urin } \\
\mathrm{k}=63\end{array}$ & 6 & 11 & $\begin{array}{l}\text { Soro } \\
\kappa=1740\end{array}$ & $\begin{array}{l}\text { Urin } \\
k=51\end{array}$ & 2 \\
\hline 13 & A & 200 & 100 & 10,5 & $\beta 1,5$ & A 1070 & 8 & 10,5 & B 1,3 & A 820 & 6 \\
\hline 14 & D & 100 & ----- & 10,5 & $\gamma 4,5$ & G 4380 & 18 & 11 & $\gamma 4,8$ & G 4700 & 11 \\
\hline 15 & B & 200 & 200 & 11 & $\gamma 2,0$ & G 2500 & 3,5 & 11,5 & $\gamma 1,4$ & G 950 & 2 \\
\hline 16 & A & 200 & 50 & 10,2 & $\gamma 1,6$ & G 1700 & 10 & 10,9 & $\gamma 1,0$ & G 754 & 5 \\
\hline 17 & B & 200 & 100 & 10,2 & $\gamma 1,7$ & G 1600 & 2 & 11,2 & $\gamma 1,2$ & G 1500 & 4 \\
\hline 18 & C & 200 & 300 & 9,1 & $\beta 3,16$ & A 3021 & 40 & 11,5 & $\beta 1,60$ & A 1940 & 9 \\
\hline 19 & A & 200 & 100 & 7,7 & $\gamma 2,59$ & G 2250 & 10 & 11 & $\gamma 1,8$ & G 1570 & 5 \\
\hline 20 & $\mathrm{D}$ & 200 & 100 & 10,5 & $\gamma 5,66$ & G 4600 & 15 & 9,7 & $\gamma 4,7$ & G 4110 & 20 \\
\hline 21 & $B$ & 200 & 50 & 12,9 & & $\begin{array}{l}\text { Urin } \\
k=6,9 \\
g=3,1\end{array}$ & $5 \%$ & 13,5 & & $\begin{array}{l}\text { Urin } \\
k=6,0 \\
g=3,5\end{array}$ & $2 \%$ \\
\hline 22 & B & 200 & 100 & 12 & $\gamma 1,9$ & G 1190 & 3 & 12,8 & $\gamma 1,29$ & G 1020 & 5 \\
\hline 23 & A & 200 & 150 & 11 & B 2,1 & A 1100 & 6 & 12,5 & $\beta 1,60$ & A 940 & 5 \\
\hline 24 & B & 200 & 100 & 13 & Não Sec & & 2 & 14 & & & 4 \\
\hline 25 & C & 200 & 100 & 8,5 & $\beta 2,9$ & A 2670 & 10 & 10,5 & $\beta 2,0$ & A 1900 & 7,5 \\
\hline 26 & C & 200 & 100 & 7,7 & $\beta 2,1$ & A 1100 & 6 & 12,5 & $\beta 2,60$ & A 1040 & 5 \\
\hline 27 & B & 200 & 200 & 10,2 & $\gamma 1,29$ & G 1080 & 3 & 12,3 & $\gamma 1,19$ & G 1200 & 5 \\
\hline 28 & D & 200 & 100 & 9,2 & $\gamma 3,9$ & G 6000 & 43 & 10,8 & $\gamma 1,16$ & G 1180 & 7 \\
\hline 29 & B & 200 & 100 & 10,9 & $\gamma 1,08$ & G 1210 & 4 & 12,2 & $\gamma 1,09$ & G 1300 & 5 \\
\hline 30 & B & 200 & 200 & 10,6 & $\gamma 1,9$ & G 2050 & 11 & 11,9 & $\gamma 1,5$ & G 1800 & 5 \\
\hline 31 & B & 200 & 200 & 11 & $\gamma 0,82$ & G 1500 & 1 & 13 & $\gamma 0,96$ & G 1450 & 2 \\
\hline 32 & E & 200 & 150 & 9 & $\gamma 4,12$ & G 4240 & 35 & 10,9 & $\gamma 2,1$ & G 1950 & 10 \\
\hline 33 & B & 200 & 100 & 12 & $\gamma 1,01$ & G 1390 & 2 & 13,8 & $\gamma 0,89$ & G 1590 & 5 \\
\hline 34 & $\mathrm{D}$ & 100 & 50 & 6,8 & $\gamma 3,75$ & G 4800 & 29 & 7,9 & $\gamma 3,46$ & G 3080 & 15 \\
\hline 35 & $E$ & 100 & 100 & 7,3 & & $\begin{array}{l}\text { Urin } \\
\kappa=7,9 \\
\lambda=3,1\end{array}$ & 31 & 7,0 & & $\begin{array}{l}\text { Urin } \\
\mathrm{k}=8,9 \\
\mathrm{~g}=4,5\end{array}$ & 22 \\
\hline
\end{tabular}

* Ind= Indicação do uso: $A$ = pós-químio de indução; $B=$ manutenção plateau pós-tmo autólogo; $C$ = recaída após esquemas químio; $D=$ refratariedade; $E=$ indução $1^{a}$ linha; ** pico $m=$ parâmetro para avaliação de resposta conforme critério de Bladé

ramide), contendo um átomo de carbono. Foi sintetizada pela vez em 1953, na Alemanha Ocidental. Inicialmente testada em animais sem demonstrar prejuízos, mostrou propriedades sedativa, hipnótica e antiemética. Entre 1957 e 1960, foi muito usada para os enjôos matinais das grávidas. ${ }^{21,25} \mathrm{O}$ preço acessível e a falta de fiscalização ocasionaram seu uso abusivo, em dose além da testada pelos cientistas, permitindo que os efeitos danosos fossem reconhecidos, destacando-se a neuropatia e a teratogenicidade estampada em mais de 10.000 bebês que nasceram com defeitos congênitos entre os anos de 1958-61, ano em que a Food and Drug Administration (FDA), nos
Estados Unidos, baniu a talidomida do mercado. ${ }^{26}$ Poucos anos depois, em 1965, uma nova indicação foi descoberta casualmente pelo dermatologista israelense, Dr. Sheskin, que usou a talidomida para sedar os pacientes com lesões dolorosas causadas pelo eritema nodoso hansênico e, surpreendentemente, as lesões inflamatórias regrediram rapidamente, mantendo a inflamação sob controle. ${ }^{27,28,29}$ Desde então, a ação antiinflamatória e imunomoduladora da talidomida começou a ser explorada. Ressurgiu neste período como tratamento das dermatoses inflamatórias crônicas, incluindo as artrites e lupus. ${ }^{26,30}$ Nos anos 80, passou a ser um recurso na principal com- 
plicação pós-transplante alogênico de medula óssea, a Doença do Enxerto contra Hospedeiro (DECH), um processo imunomediado, envolvendo o linfócito T, com manifestações mucocutâneas, que podem ser controladas pelo seu efeito supressor sobre o fator de necrose tumoral alfa. ${ }^{31,32}$

Desde a sua reintrodução muitas evidências foram divulgadas:

1984 - Barnhill RL et al ${ }^{33}$ referiram a capacidade da talidomida em inibir a fagocitose de leucócitos em culturas, podendo justificar seu efeito antiinflamatório.

1991- Sampaio EP et al ${ }^{34}$ demonstraram a ação inibitória sobre o fator de necrose tumoral alfa (alfa TNF) em monócitos humanos "in vitro", abrindo caminho para seu uso em situações que cursam com caquexia, como as doenças consuptivas.

1993 - Makonnnkawkeyyoon $\mathrm{S}$ et $\mathrm{al}^{35}$ publicaram os resultados de suas investigações, afirmando que a talidomida pode impedir a replicação do vírus da Imunodeficiência Humana Tipo 1 (HIV 1) nos monócitos de pessoas infectadas. Outros autores (RJ Powell -BMJ, 1996) acreditam que a talidomida não inibe a replicação viral do HIV, mas age nos aidéticos revertendo a caquexia provocada pelo vírus.

1994 - D’ Amato RJ et a ${ }^{36}$ fizeram observações sobre a angiogênese, onde a talidomida exerceu efeito antivasculogênese em córnea de coelho. A partir desta pesquisa, foi sugerido o uso da talidomida nas doenças como retinopatia diabética e tumores sólidos.

1995 - Mchuhg S et al ${ }^{37}$ comunicaram o efeito singular em converter a resposta linfocitária antigênica dos $\mathrm{T}$ helper 1 para T helper 2, in vitro. Se este efeito for comprovado em humanos, a talidomida pode ter uma nova atuação em doenças que provocam o desequilíbrio entre as populações de CD4/CD8.

1996 - Neubert $\mathrm{R}$ et $\mathrm{al}^{38}$ apresentaram resultados em macacos, comprovando a propriedade imunomoduladora da talidomida, mostrando que ela regula moléculas de adesão celular e induz mudanças no padrão das integrinas.

1999 - Singhal S et al ${ }^{7}$ reportaram os primeiros resultados dos estudos com a talidomida, iniciados em 1997, em pacientes com Mieloma Múltiplo. Os resultados animadores sustentam as hipóteses de um efeito antiangiogênico e imunomodulador.

A partir de 1999, inúmeras publicações internacionais têm divulgado o benefício da talidomida no MM, passando esta a fazer parte dos recursos terapêuticos em questão.

\section{Farmacologia}

A talidomida farmacologicamente ainda representa um enigma. É um agente imunomodulatório com um espectro de ação que até hoje ainda não está completamente dissecado pela falha de um conhecimento básico de seu mecanismo de ação, de seus metabólitos e de suas concentrações plasmáticas. ${ }^{39}$ Embora seu metabolismo não seja hepático, uma quantidade desta droga é metabolizada pelas enzimas do citocromo $\mathrm{P} 450 .{ }^{21}$ Sua principal via de degradação é uma clivagem hidrolítica no plasma e outros fluidos corporais, degradando-se em vários metabólitos com excreção de uma pequena quantidade pela urina. $\mathrm{O}$ clearence renal é de $1,15 \mathrm{ml} / \mathrm{min}$ e menos de $0,7 \%$ da dose é eliminada intacta na urina. ${ }^{21,39} \mathrm{~A}$ biodisponibilidade da via oral é alta e os alimentos não afetam a absorção ou os picos plasmáticos de concentração. ${ }^{21,40} \mathrm{~A}$ absorção pelo trato gastrointestinal é lenta, levando cerca de duas horas para atingir concentração plasmática máxima e mantendo uma meia vida de cinco a sete horas. Recentemente, sua utilidade clínica tem sido ampliada com base no seu efeito imunomodulatório. A chave para sua atividade biológica está na sua capacidade de: ${ }^{20,41,42}$

1) modular a produção monocitária do fator de necrose tumoral $\alpha$ (TNF), das IL-6, IL-1 $\beta$ e IL-10, citocinas indispensáveis para o crescimento da células do microambiente e do tumor;

2) modular a expressão das moléculas de adesão: ICAM-1(CD54), VCAM-1(CD106), E-seletinas e Lseletinas, integrinas de interface entre o tumor e o microambiente;

3) promover a apoptose;

4) propriedade antiangiogênica, inibindo os fatores de crescimento do endotélio vascular e o fator de crescimento de fibroblastos;

5) indução da produção de citocinas moduladoras do tumor, como o interferon $\gamma$ e a IL-2 ; de CD8.

6) potencializa a ação dos linfócitos $T$, basicamente

\section{Aplicações clínicas}

A partir do seu ressurgimento, a talidomida contemplou diversas entidades clínicas. Nos meados da década de 60, as dermatopatias encabeçaram a lista, com índices de resposta próximos a $90 \%$ de remissão. ${ }^{28}$ Nos anos seguintes sua aplicação foi abrangendo novas doenças, iniciando com as reumatopatias de cunho imune, seguidas pelas Doença do Enxerto contra o Hospedeiro, Síndrome de Imunodeficiência Adquirida (SIDA), e, no final dos anos 90, com as neoplasias hematológicas e posteriormente os tumores sólidos. ${ }^{20,43,44,45}$ As tabelas 12 e 13 informam as respostas obtidas com a talidomida nas doenças inicialmente preconizadas e nas doenças hematológicas respectivamente. $^{20}$

\section{Efeitos colaterais}

$\mathrm{O}$ efeito devastador da talidomida é a deformidade em fetos, incluindo defeitos cardíacos, renais, genitais e a focomelia, todos secundários ao fechamento precoce da vascularização nestes órgãos, efeito hoje conhecido como 
Tabela 12

Aplicação da talidomida em doenças inflamatórias

\begin{tabular}{lccc}
\hline Doença & $N^{\circ}$ de casos & Remissões & $\%$ \\
\hline Aftose oral & 113 & 81 & $71 \%$ \\
Doença de Beçhet & 66 & 54 & $81 \%$ \\
Lupus discóide & 112 & 101 & $91 \%$ \\
Eritema polimorfo & 5 & 5 & $100 \%$ \\
Prurigo actínico & 9 & 8 & $88 \%$ \\
Pioderma gangrenoso & 4 & 4 & $100 \%$ \\
Úlceras aftosas no HIV+ & 62 & 51 & $82 \%$ \\
Doença do Enxerto & & & $50 \%$ \\
$\quad$ Versus Hosp & 6 & 3 & \\
\hline
\end{tabular}

Fonte: Calderon P, Anziolotit M, Phelps R. "Thalidomide in Dermatology: New Indications for Old Drug". Intern Journ Dermat 1997;36:881-887

Tabela 13

Aplicação da talidomida nas hematopatias

\begin{tabular}{lcc}
\hline Doença & $\begin{array}{c}\text { No }^{\circ} \\
\text { pacientes }\end{array}$ & $\begin{array}{c}\text { Média } \\
\text { remissões (\%) }\end{array}$ \\
\hline Mieloma Múltiplo & 664 & $35 \%$ \\
Macroglobulinemia Wald & 20 & $20 \%$ \\
Amiloidose primária & 12 & $41 \%$ \\
Mielodisplasia & 83 & $31 \%$ \\
Mielofibrose & 15 & $43 \%$ \\
Linfoma não-Hodgkin & 12 & $08 \%$ \\
\hline
\end{tabular}

Fonte: Kumar S, Witzig TE, Rajkumar V. Thalidomide as an anticancer agent. JCMM 2002; 1582-1594

antiangiogênico, considerado útil para bloquear a sustentação das células tumorais que dependem da neovascularização. ${ }^{46,47}$ A única contra-indicação absoluta desta droga é a gestação. A talidomida não apresenta toxicidade aguda, ${ }^{21}$ no entanto, os paraefeitos estão relacionados ao acúmulo de doses. Após duas a três semanas, alguns desconfortos podem ser atribuídos ao seu uso. A neuropatia periférica é provavelmente o paraefeito mais limitante, e geralmente manifesta-se após doses cumulativas que podem variar de $6 \mathrm{~g}(60 \mathrm{cp})$ até $50 \mathrm{~g}(500 \mathrm{cp})$ em alguns pacientes. ${ }^{48} \mathrm{~A}$ talidomida induz a neuropatia sensorial mais proeminente que motora através da redução das fibras mielinizadas. Sonolência, relaxamento da musculatura e supressão dos reflexos ocorrem com relativa freqüência. Hipersensibilidade cutânea pode ser percebida precocemente, a partir de dois dias de introdução e deve ser controlada com anti-histamínicos. A constipação ocorre em $1 / 3$ dos pacientes e usualmente resolve com reeducação de dieta e/ou emolientes. Os efeitos hematológicos como leucopenia e plaquetopenia podem estar mais associados ao vírus do HIV e revertem rapidamente com a interrupção temporária da droga. Eventos tromboembólicos são descritos em $3 \%$ a $28 \%$, principalmente quando a talidomida é usada em associação com dexametasona ou poliquimioterapia. Na fase aguda das tromboses é prudente sus- pender a droga, entretanto, após a anticoagulação adequada é possível recomeçar a talidomida. São muitos os efeitos colaterais descritos como decorrentes da talidomida e estão expostos no quadro 1 de acordo com a freqüência dos órgãos e sistemas acometidos. ${ }^{48}$ Todos estes são dose dependentes, podendo reverter completamente após redução da dosagem ou retirada da droga. ${ }^{7}$

Quadro 1

Efeitos colaterais classificados pela freqüência e sistemas

\begin{tabular}{lc}
\hline Classificação por Sistemas & Freqüência \\
\hline 1. Reações Centrais & \\
Sonolência & $37 \%$ \\
Vertigem & $20 \%$ \\
Parestesias & $15 \%$ \\
Agitação & $9,5 \%$ \\
Neuropatia periférica & $8,5 \%$ \\
Cefaléia & $4 \%$ \\
Redução da libido & $4 \%$ \\
Tremores & $<1 \%$ \\
Alteração do humor & $<1 \%$ \\
Enxaqueca & $<1 \%$ \\
Euforia & $<1 \%$ \\
Depressão & $<1 \%$ \\
2. Reações Hematológicas & \\
Leucopenia & $25 \%$ \\
Anemia & $12,5 \%$ \\
Plaquetopenia & $11 \%$ \\
Linfoadenopatias & $10 \%$ \\
Pancitopenia & $5 \%$
\end{tabular}

3. Reações Dermatológicas

Rash cutâneo

$12,5 \%$

Ressecamento pele $\quad 10 \%$

Acne

Dermatite fúngica

Alterações da unha

Alopécia

Eczantema

Urticária

Prurido

$7 \%$

$6,5 \%$

$4 \%$

$3 \%$

$<1 \%$

$<1 \%$

$<1 \%$

4. Reações Gastrointestinais

Naúseas

$12,5 \%$

Secura da boca

$10 \%$

Monilíase oral

$8 \%$

Flatulência

Constipação

Aumento TGO

Aumento de bilirubinas

Distúrbio do paladar

Aumento da parótida

Distensão do abdome

Colite

$8 \%$

$8 \%$

$7,5 \%$

$<1 \%$

$<1 \%$

$<1 \%$

$<1 \%$

$<1 \%$

5. Reações Respiratórias

Faringites

Sinusopatias

Tosse

Alteração da voz

Bronquite

$6 \%$

$5 \%$

$<1 \%$

$<1 \%$

$<1 \%$

6. Reações Endocrinológicas e Metabólicas

$$
\begin{aligned}
& \text { Hiperlipemia } \\
& \text { Edema periférico } \\
& \text { Hiperuricemia }
\end{aligned}
$$

$7 \%$
$5,5 \%$

$<1 \%$ 
Hipomagnesemia

Hipercalemia

Hipocalcemia

Hipocolesterolemia

Ginecomastia

Hipo ou hiperglicemia

Hipotireoidismo

Distúrbios menstruais

7. Reações Cardiovasculares

Tromboses

Arritmias

Hipertensão

Hipotensão postura

Distúrbios vasculares periféricos

Palpitações

Fenômeno de Raynaud

8. Reações Oftalmológicas

Diplopia

Dor ocular

Redução da lágrima

Conjuntivite

Retinite

$<1 \%$

$<1 \%$

$<1 \%$

$<1 \%$

$<1 \%$

$<1 \%$

$<1 \%$

$<1 \%$

3 a $28 \%$

$<1 \%$

$<1 \%$

$<1 \%$

$<1 \%$

$<1 \%$

$<1 \%$

$<1 \%$

$<1 \%$

$<1 \%$

$<1 \%$

$<1 \%$

Quadro 2

Medidas mitigadoras para os efeitos da talidomida

\begin{tabular}{|c|c|}
\hline Efeito colateral & Medidas recomendadas \\
\hline Sonolência & $\begin{array}{l}\text { Tomar a talidomida na hora de dormir; } \\
\text { Evitar uso de bebidas alcoólicas; } \\
\text { Evitar outras medicações que } \\
\text { possam contribuir para sonolência; } \\
\text { Evitar tarefas que necessitem } \\
\text { atenção e reflexos, tipo dirigir }\end{array}$ \\
\hline Neuropatia periférica & $\begin{array}{c}\text { Caminhadas e outros exercícios leves } \\
\text { Evitar meias elásticas; } \\
\text { Exames neurológicos periódicos a } \\
\text { cada } 3 \text { meses; } \\
\text { Redução da dose de talidomida; } \\
\text { Interrupções temporárias da } \\
\text { talidomida; } \\
\text { Medicações tipo complexos } \\
\text { vitaminas B }\end{array}$ \\
\hline Tonturas & $\begin{array}{l}\text { Esperar alguns minutos deitado } \\
\text { após a ingesta da talidomida }\end{array}$ \\
\hline Constipação & $\begin{array}{l}\text { Aumentar ingesta diária de água } \\
\text { (8-10 copos/dia); } \\
\text { Dieta rica em fibras; } \\
\text { Exercícios físicos e caminhadas; } \\
\text { Usar emolientes fecais ou laxantes }\end{array}$ \\
\hline Rash cutâneo & $\begin{array}{l}\text { Uso de anti-histamínicos; } \\
\text { Cremes e loções tópicas } \\
\text { à base de manteiga de cacau, } \\
\text { aveia ou calêndula } \\
\text { Sabonetes de aveia }\end{array}$ \\
\hline Tromboses & $\begin{array}{l}\text { Interromper temporariamente } \\
\text { a talidomida; Anticoagulação; } \\
\text { Reintrodução lenta após } \\
\text { anticoagulação adequada }\end{array}$ \\
\hline Secura da boca & $\begin{array}{l}\text { Hidratação via oral freqüente; } \\
\text { Gel umidificante } \\
\text { (tipo produtos da Biotene) } \\
\text { Saliva artificial } \\
\text { Mascar chicletes próprios para } \\
\text { estimular a produção de saliva }\end{array}$ \\
\hline
\end{tabular}

\section{Medidas mitigadoras}

Alguns dos paraefeitos da talidomida podem ser atenuados através de simples medidas. A neuropatia, um dos efeitos mais incapacitantes, pode ser monitorizada com avaliações neurológicas e eletrofisiológicas periódicas. Se o potencial de ação de neurossensibilidade perde mais de $30 \%$ de sua amplitude, recomenda-se interromper a talidomida e reintroduzi-la quando a amplitude da neurossensibilidade retornar ao basal. ${ }^{49}$ Entretanto, algumas medidas mitigadoras, como evitar meias e sapatos apertados e suplementação com complexo vitamínico $\mathrm{B}$, podem ser benéficas e, em alguns casos, podem evitar a suspensão da medicação. Assim como na neuropatia, em outros efeitos como a constipação e rash cutâneo existem algumas medidas preventivas auxiliares descritas no quadro $2 .^{50}$

\section{Abstract}

Over the last two decades, we have seen a radical change in therapy and progression of multiple myeloma, a malignant hematologic disease that is still considered fatal. Recent investment and research on mechanisms that interfere in the physiopathogenesis and bone marrow microenvironment are turning control and regression of the malignant plasma cell clone into something achievable, which may change expectations related to this disease. The new idea of using an old drug, thalidomide, has shown to be effective in multiple myeloma. In 1997, using the known effects of immunomodulation and antiangiogenesis of this drug, clinical trials were started in patients with unresponsive disease. Other therapeutic interventions in the bone marrow microenvironment and plasma cells have been added and proved to be efficacious, not only as a therapy for refractory patients, but also for induction and/or remission maintenance therapy. Thirty-five patients with multiple myeloma were treated with low-dose thalidomide $(100 \mathrm{mg})$ and followed up. Thirteen were on maintenance therapy after bone marrow transplantation, eleven started thalidomide after induction therapy, five after relapse, four were refractory to usual therapies and two had induction therapy with thalidomide. The study took place in the Hematology and Bone Marrow Transplantation service of the Hospital de Clinicas de Porto Alegre, from March 2001 to December 2003. Hemoglobin levels, serum or urine immunoglobulin peaks and bone marrow plasma cell counts were evaluated. These parameters were assessed before starting with the drug and after 3.6 and 12 months of usage. The immunoglobulin level was considered the gold standard to evaluate the response. The results showed that $100 \mathrm{mg}$ was the tolerable dose for $51 \%$ of the patients. Sixty-five percent of those who used thalidomide for induction therapy showed a 25 to $50 \%$ improvement in immunoglobulin serum levels and 90\% of the patients on maintenance therapy (13 after bone marrow transplantation, 11 after induction), sustained the same immunoglobulin levels of the initial plateau. Rev. bras. hematol. hemoter. 2004;26(4):245-255.

Key words: Thalidomide; multiple myeloma; anti-angiogenesis. 


\section{Referências Bibliográficas}

1. Foerster J Paraskevas F. Multiple Myeloma. In: Lee R, Foerster J, Lukens J et al. Wintrobe's Clinical Hematology. Baltimore, Williams \& Wilkins. 10 ed, 1999; cap 99: 2631-2680.

2. Anderson K, Barlogie B, Alexenian R et al. Thalidomide: An emerging role in treatment of myeloma and others malignancies. A Corporate Friday Symposium The American Society of Hematology Annual Meeting, 1999: 2-14.

3. Kyle R. Multiple Myeloma and others plasma cell disorders. In: Hoffman R, Benz EJ, Shattil SJ et al. Hematology - Basic Principles and Practice. 2nd ed. New York: Churchill Livingstone, 1995;1354-74.

4. Anderson K, Kyle AR, Berenson J. Multiple Myeloma. Education Program: The American Society of Hematology Meeting, 1997:177-188.

5. Kyle R. Newer approaches to the management of Multiple Myeloma. Cancer Supplement 1993;72(11):3.489-3.494.

6. Bladé J, Samson D, Reece D et al. On behalf of Myeloma subcommittee of the EBMT, Chronic Leukemia Working Party and the Myeloma Working Committee of the IBMTR. Br J Haematol. 1998;102(5):1115-1123.

7. Singhal S, Mehta J, Desikan R et al. Antitumor activity of thalidomide in refractory multiple myeloma. N Engl J Med 1999;341:1.565-71.

8. Kneller A, Raanani P, Hardan I et al. Therapy with thalidomide in refractory multiple myeloma patients -the revival of an old drug. Br J Haematol 2000;108:391-393.

9. Rajkumar SV, Gertz MA, Kyle RA, Greipp Pr. Current therapy for multiple myeloma. Mayo Clin Proc 2002;77:813-822.

10. Weber D, Rankin K, Galvino $M$ et al. Thalidomide alone or with dexamethasone for previously untreated multiple myeloma. J Clin Oncol 2003;21(1):16-19.

11. Rajkumar SV, Gertz MA, Lacy MQ et al. Thalidomide as initial therapy for early-stage myeloma. Leukemia 2003;17(4):775779.

12. Vacca A, Ribatti D, Presta M et al. Bone marrow neovascularization, plasma cell angiogenic potential, and matrix metalloproteinase2 secretion parallel progression of human multiple myeloma. Blood 1999;93(9):3.064-3.073.

13. Vacca A, Loreto MD, Ribatti D et al: Bone marrow of patients with active multiple myeloma: Angiogenesis and plasma cell adhesion molecules LFA-1, VLA-4, LAM-1 and CD 44. Am J Hematol 1995;50:9-14.

14. Barlogie B, Desikan R, Eddlemon P et al. Extended survival in advanced and refractory multiple myeloma after single-agent thalidomide: identification of prognostic factors in a phase 2 study of 169 patients. Blood 2001;98(2):492-494.

15. Juliusson G, Celsing F, Turesson I et al. Frequent good partial remissions from thalidomide including best response ever in patients with advanced refractory and relapsed myeloma. $\mathrm{Br} \mathrm{J}$ Hematol 2000;109:89-96.

16. Tosi P, Ronconi S, Zamagni E et al. Salvage therapy with thalidomide in multiple myeloma patients relapsing after autologous peripheral blood stem cell transplantation. Haematologica 2001;86:409-413.

17. Barlogie B, Zangari M, Spencer T, et al. Thalidomide in the management of multiple myeloma. Semin Hematol 2001;98: 3.846-3.848.

18. Larkim M. Low-dose Thalidomide seems to be effective in multiple myeloma. Lancet 1999;354:925.
19. Palumbo A, Giaccone L, Bertola A et al. Low-dose thalidomide plus dexamethasone is an effective salvage therapy for advanced myeloma. Haematologica 2001;86:399-403.

20. Kumar S, Witzig Te, Rajkumar V. Thalidomide as an anticancer agent. JCMM 2002;1582-1594.

21. Perri Aj, Hsu S. A review of thalidomide's history and current dermatological applications. Dermatology Online Journal 2003 $9(3): 5$.

22. Kumar S, Gertz MA, Dispenzieri A et al. Response rate, durability of response, and survival after thalidomide therapy for relapsed multiple myeloma. Mayo Clin Proc 2003;78:34-39.

23. Neben K, Moehler T, Benner A et al. Dose-dependent effect of thalidomide on overall survival in relapsed Multiple Myeloma. Clinical Cancer Research 2002;8:3.377-82.

24. Durie B, Stepan D. Efficacy of low dose thalidomide in multiple myeloma. Eur J Oncol 2000;1:1-8.

25. Duarte F. Question: What is Thalidomide. Chemistry 1998;1: $1-2$.

26. Sato EI, Assis LS, Lourezi VP, Andrade LEC. Long-term Thalidomide use in refractory cutaneous lesions of systemic lupus erythematosus. Rev Ass Med Brasil 1998;44(4):289-93.

27. Tseng S, Pak G, Washenik et al. Rediscovering thalidomide: A review of its mechanism of action, side effects, and potential uses. J Am Acad Dermatol. 1996;35(6):969-79.

28. Calderon P, Anziolotti M, Phelps R. Thalidomide in dermatology: New indications for old drug. Intern Journ Dermat 1997;36: 881-887.

29. Powell RJ. Thalidomide: Currents uses. Bio Drugs 1999;11: 409-416.

30. Cwyynarski K. Old drugs and new approaches to haematology. The Lancet 1998;352:1992.

31. Lim SH, Mcwhannell A, Vora AJ, Boughhton BJ. Successful treatment with thalidomide of acute graft-versus-host disease after bone marrow transplantation. The Lancet 1988;117.

32. Heney D, Norfolk DR, Wheeldon J et al. Thalidomide treatment for graft-versus-host disease. British Journ Haematol 1991; 78:23-27.

33. Barnhill RL, Doll NL, Millikan LE, Hastings RC. Studies on the antiinflammatory properties of thalidomide. Ach Dermatol Res 1980;269:275-280.

34. Sampaio EP, Sarno EM, Galilly R et al. Thalidomide selectively inhibits tumor necrosis factor alpha production by stimulated human monocytes. J Exp Med 1991;173:699-703.

35. Makonkawkeyoon S, Limson-Pobre RNR, Moreira AL et al. Thalidomide inhibits the replication of human immunodeficiency virus type I. Proc Natl Acad Sci USA 1993;90:5.974-5.978.

36. D’Amato RJ, Lougnan MS, Flynn E, Folkman J. Thalidomide is inhibitor of angiogenesis. Proc Natl Acad Sci USA 1994;91: 4.082-4.085.

37. Mchugh SM, Rifkin IR, Deigton J et al. The immunosuppressive drug thalidomide induces $\mathrm{T}$ helper cell type 2 (Th2) and concomitantly inhibits Th1 production in mitogen- and antigen-stimulated human peripheral blood mononuclear cell cultures. Clin Exp Immunol 1995;99:160-167.

38. Neubert R, Hinz N, Thiel R, Neubert D. Down regulation of adhesion receptors on cells of primate embryos as a probable mechanism of teratogenic action of thalidomide. Life Sci 19951996;58:337-348

39. Eriksson T, Bjorkman S, Hoglund P. Clinical pharmacology of thalidomide. Eur J Clin Pharmacol 2001;57(5):365-76. 
40. Radorasky CL, Levine N. Thalidomide. Dermatol Clinics 2001; 19(1):87-103.

41. Noopur R, Anderson K. Thalidomide - A Revival Story. The New Engl Journ Med 1999;341(21):1.606-1.608.

42. Geitz H, Handt S, Zwingenberger K. Thalidomide selectively modulates the density of cell surface molecules involved in the adhesion cascade. Immunopharmacology 1996;31:213

43. Dimopoulos MA, Zomas A, Viniou NA et al. Treatment of Waldrenstrom's macroglobulinemia with thalidomide. J Clin Oncol 2001;19:3.596.

44. Seldin DC, Choufani E, Skinner M et al. A phase I/II trial of thalidomide for patients with AL amyloidosis. Blood 2001;98: abstract 691 .

45. Figg WD, Dahut W, Duray $P$ et al. A randomized phase II trial of thalidomide, an angiogenesis inhibitor, in patients with androgenindependent prostate cancer. Clin Cancer Res 2001; $7: 1.888$.

46. Brown K. New uses for thalidomide yielding valuable lessons. The Scientist 1997;11(3):1-8.

47. Barlogie B. Thalidomide in multiple myeloma. The New Engl Journ Med 2000;342(13):975-976.

48. Ochonisky S, Verroust J, Bastuji-Garin S et al. Thalidomide neuropathy incidence and clinico-electrophysiologic findings in 42 patients. Arch Dermatol 1994;130(1):66-9.

49. Powell RJ, Gardner-Medwin JMM. Guideline for the clinical use and dispensing of thalidomide. Postgrad Med J 1994;79:901904.

50. International Myeloma Foundation. Understanding Thalidomide Therapy. Patient Handbook. 2003.

Avaliação: Editor e dois revisores externos

Conflito de interesse: não declarado

Recebido: 02/08/2004

Aceito após modificações: 28/08/2004 